\title{
Designing Enjoyable Multimodal Activities to Reduce Falls Risk in the Community
}

\author{
Stephen Uzor \\ Multimodal Research Group \\ School of Engineering and Computing \\ Glasgow Caledonian University \\ Glasgow, UK \\ stephen.uzor@gcal.ac.uk
}

\author{
Lynne Baillie \\ Multimodal Research Group \\ School of Engineering and Computing \\ Glasgow Caledonian University \\ Glasgow, UK \\ 1.baillie@gcu.ac.uk
}

\author{
Dawn Skelton \\ School of Health \\ Glasgow Caledonian University \\ Glasgow, UK \\ dawn.skelton@gcu.ac.uk
}

\begin{abstract}
This paper summarizes on-going work aimed at improving falls rehabilitation in the community using enjoyable multimodal exercise games. There have been numerous successful trials involving the use of exercise interventions to reduce falls risk. However, there is an existing problem with adherence to these exercises in the home. We propose the use of games - based on established programmes of exercise for falls prevention - to encourage independence and motivation to exercise in community-dwelling older adults at risk of falls. Current evidence shows that games can be used to aid rehabilitation; with most studies focusing on the use of commercially available games. In cases where certain limb motions are required for effective recovery; special games and hardware tools that encourage these motions are needed. Because such tools are often expensive and complex to set up; most interventions of this type have been restricted to the laboratory setting. In order to deliver these activities to community-dwelling users, we investigated the use of various portable wireless technologies to help achieve this aim. Finally, we describe the next stage of our research in which we intend to co-design with falls patients, engaging multimodal activities to motivate users to exercise. Once this phase is complete, we will evaluate, through trials, the use of these activities in the home.
\end{abstract}

Keywords - falls; rehabilitation; home exercise; RCT; design workshop; user interaction

\section{INTRODUCTION}

According to [9], 30\% of people over the age of 65 fall at least once a year with over $75 \%$ of this population suffering an injury as a result. Fractures (the leading consequence of falls) account for a large percentage of hospital admissions and can result in a loss of independence, fear of falling and even death in older adults. Although falls can be caused by a wide variety of factors (including diseases and physical impairments); many cases are attributed to a lack of exercise, which can lead to weaknesses in muscles and joints in the lower body [1].

These conditions can be alleviated by rehabilitation designed to improve the patient's ability to cope with movement in everyday life, increase the patient's functional capacity; elevate confidence and reduce the fear of falling [15].

\section{A. Exercise Interventions for Falls}

Evidence has shown that certain programmes of exercise such as the Falls Management Exercise (FaME) and Otago Exercise (OEP) programmes are effective in returning falls patients to normal functional movement (if the patient adheres to the routine) and can reduce the risk of falling by as much as $30 \%[16]$. However, there is a problem with adherence to these exercises in the community which means that these interventions are often ineffective when used in this setting.

In order to investigate the reasons behind these low adherence rates, we spoke to experts who had conducted many trials involving exercise interventions for falls prevention in the community. They reported that there seemed to be a major problem with the modes of delivery of these exercise programmes - using DVDs and paper-based illustrations. This meant that, due to the essential repetitive nature of these exercises [10], the rehabilitation process often felt like a chore for the subjects.

Taking this into account, we identified an important research question:

- How can we distract the user from the monotonous nature of these important exercises; provide a more enjoyable experience and still promote the advantages of repetitive motion required for effective falls prevention in the community?

We believe that one way of doing this is by providing engaging multimodal activities - that are appropriate to the level of interaction required for rehabilitation - to motivate users to exercise.

Medical Research Council LLHW Initiative 


\section{B. Games for Rehabilitation}

Exercise games have been successfully used in previous research to aid rehabilitation, but the majority of these studies have focused on stroke rehabilitation (mainly to support recovery of motor function in affected limbs). There have been a number of falls prevention studies involving the use of games to motivate users to exercise [5][6]. Nevertheless, most of these interventions have been restricted to the laboratory because of the complex nature - and modes of operation - of equipment used for the delivery of these games.

A preliminary study conducted by the makers of the SilverFit softkinetic rehabilitation system revealed that the participants often performed more exercise than was required of them as they found most of the games very addictive [14]. The system utilised a large screen and a depth sensing camera capable of translating natural human movement into on-screen actions. Even though this type of depth sensing camera technology is very accurate; it is bulky, expensive and often requires a professional to initialise and operate it.

Because our research is concerned with the prevention of falls in the community setting; it was necessary to investigate the use of alternative, portable low-cost technologies for the delivery of these exercise games in the home.

\section{AIMS AND OBJECTIVES}

The main aim of our research involves the design and development of engaging multimodal activities to aid rehabilitation in the community. Because there is an emphasis on motivation and encouraging independence; it is imperative that these multimodal activities conform to a certain aesthetic and functionality that will enable effective interaction by the users. Bearing this in mind, we identified two major objectives for this study:

- To involve users at risk of falling in the design of conceptual games based on established programmes of exercise.

- Evaluate the effectiveness of the aforementioned multimodal games on increasing adherence to exercise in the community.

\section{RESEARCH PROTOCOL}

To satisfy the objectives outlined above, our research will involve a number of trials utilising a variety of hardware and software tools for the delivery of exercise games in the home. To address the issues with the various topics involved in this research (such as - software platforms, adherence, system design, etc.), it is important that we use a multi-method approach. This will involve a number of quantitative, as well as qualitative methods and procedures dealing with the design and evaluation components of the study.

\section{A. Qualitative Methods}

The majority of research conducted in the field of Virtual Environments (VE) has concentrated on the visual aesthetics rather than usability. The user interaction components of such VEs are often poorly designed and seldom evaluated by users and as such, many tools designed for the generation of these VEs are difficult to use [7]. The needs of the elderly often differ from those of younger people when technology is concerned and it is important that they are involved in the design process [13]. To this end, we intend to involve the users in the design of the multimodal activities mentioned in the objectives as it is crucial that the use of these tools in the community is not difficult for the user.

By involving the users in the design of the software tools, we can explore a variety of experiences, preferences and issues concerning the use of such tools in the home. This information can be used to modify the tools in order to enhance the experience for the user. Qualitative methods of relevance to this study include the following:

1) Focus Groups: The aim of the focus group is to involve users in active discussion of past experiences with rehabilitation and motivation to exercise. Through this, we hope to acquire a wide range of opinions regarding possible aesthetic and understanding issues concerning the use of interactive media during rehabilitation.

2) Design Workshops: The design workshop will involve older adults in the design of engaging activities using methods shown in previous research to enhance the user-design process [3][12]. These will include:

a) Scenarios: Scenarios are useful in conceptualizing and managing the performance of tasks in certain environments which could improve the usefulness and usability of technology [4]. Users will be asked to discuss the issues and challenges that they could face while performing exercises using booklets and DVDs (the standard rehabilitation tools used in the home).

b) User Sketches: Previous research has shown that inviting users (both children and adults) to conceptualize their ideas using sketches and drawings has encouraged greater participation in design [2]. During the design workshop, participants will be asked to design their own games in groups.

By involving the participants in this way, we hope to get an idea of the elements of our design that the participants are sceptical about; and what preferences they have regarding the appearances of these visual elements that would enhance their interaction with the system. Through this process, we can also potentially identify commonality in design ideas between participants (or groups) as the dominant preferences and concerns shared by the users could become apparent.

\section{B. Quantitative Methods}

The information gathered from the design workshops and focus groups can possibly assist in the development of a reliable system that can be deployed in a wide variety of settings. The quantitative methods of interest to this research include pilot studies and a community-based randomized controlled trial (RCT). 
1) Pilot studies: A pilot study will be carried out to assess the feasibility of using our chosen software and hardware tools in the community. The results from this study will enable us to modify these tools in order to reduce (if not eliminate) possible barriers to the users' effective interaction with the exercise games.

2) Community-Based RCT: This will be the main trial for this research. It will be conducted during a 3-month period, with the major outcomes taken at the start and at the end of the trial. Our participants will include older adults - over the age of 65 - identified as at risk of falls; living in the community and able to perform home exercise. The outcomes for our RCT are consistent with useful relevant studies in falls prevention [8][11]. They include biomechanical measures of balance, muscle strength and gait; as well as the use of questionnairebased health and quality of life evaluation tools which provide a multi-factorial assessment of a person's falls risk.

In particular, the following outcomes are of interest to our research:

- Primary Outcomes: Adherence; Balance performance using Centre of Pressure path displacement.

- Secondary Outcomes: Gait performance (gait speed, variability in stride length, stride time and double support time); quadriceps strength; falls efficacy scale (FES-I); and SF-12.

\section{TOOLS INVESTIGATION}

One of the major challenges for this research involves the effective interaction with computer-based applications by elderly users in the home. These applications include a variety of multimodal activities which will encourage body movement; in accordance with effective routines employed by established programmes of exercise for falls prevention.

It is vital to utilise a multi-factorial approach to falls prevention during rehabilitation as older adults at risk of falls are often affected by multiple falls risk factors. Our research is concerned with improving adherence to balance and strength training exercises, which we believe - from previous successful trials - could increase confidence and mobility; reduce fear of falling and encourage independence in older adults.

To explore the feasibility of using commercially available software and hardware tools to answer our research question; we developed simple prototype exercise games based on the OEP balance and strength training exercises. These games were tested with a number of hardware tools to investigate the practical issues associated with each of the different modalities. The procedures and related tests are outlined below:

\section{A. Fire Rescue Game using InfraRed (IR)Transmitters}

This was the first game we developed. It involved the use of an IR camera and two IR transmitters strapped to a user's leg as shown in Fig. 2. The objective of the game was to rescue in- game characters from a burning building using a movable platform, which was controlled by the flexion and extension motions of the user's knee. This game was based on the OEP exercise shown in Fig 1.

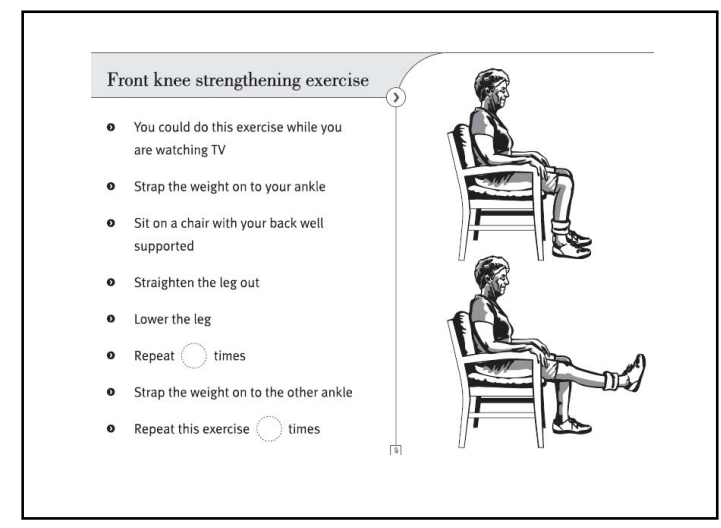

Figure 1. Illustration of the OEP "Knee Lift" exercise as used in Falls rehabilitation in the UK.

The game was demonstrated to a variety of health professionals, including physiotherapists and occupational therapists who agreed it was a great way to involve users in rehabilitation. However, it was concluded that while the sensors were non-intrusive and easy to use; they didn't work as well as we expected for the following reasons:

- The user had to make multiple adjustments to their position so that the IR transmitters could be aligned and detected by the camera.

- Reflections from other IR (or reflective) sources sometimes interfered with the transmitter signals and this affected the controls in the game.

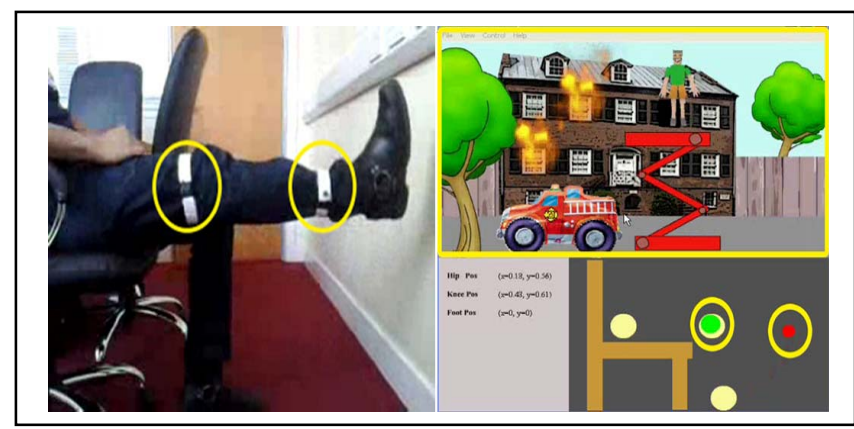

Figure 2. A user playing the "Fire Rescue" game with IR transmitters (shown in yellow circles).

\section{B. Feline Stalker Game using the Nintendo Wii Balance Board (WBB)}

We developed this game to help with balance re-training (necessary to improve balance coordination in some cases involving stroke survivors, who could also be at risk of falling). The control modality involved in this game challenged balance 
by having the user control game elements on the screen using weight distribution (calculated by the WBB). This weight distribution was translated to control the movement of a cat in the game where the aim was to catch mice that came out to feed in certain areas of a room.

This game was demonstrated to a falls expert who said that it would work very well in supervised exercise classes in the community, but the WBB could potentially become a falls hazard in the home. Because our trials involve the home use of these exercise games, we wanted to develop a set of exercise games that could work well in both supervised and unsupervised sessions. We therefore decided to consider the use of inertial sensors for the next phase of development.

\section{FUTURE WORK}

Current evidence suggests that inertial sensors - small devices capable of detecting motion - can be used for gait analysis outside the laboratory [17]. This is usually done by associating the tilt angles detected by the sensor with the related joint on the human body; and imposing this tilt on a digital mannequin to show the resultant motion on a screen. In the same way, this tilt data can be used to control objects in a game using appropriate software platforms.

We intend to use this functionality to develop our next exercise game which will be based on scooter racing. The motions required for this game are similar to those for one of the FaME exercises (designed to improve strength in the quadriceps and hamstring muscles). Users will be able to control the speed of a scooter using constant flexion and extension of one knee while standing and using a chair for support.

Inertial sensors offer the advantage of being independent nodes that can send signals wirelessly to a receiver - attached to a computer - with minimum interference from other objects in the environment. Consequently, we intend to employ this technology in the development of a number of exercise games based on more OEP and FaME balance and strength training exercises. Prototype versions of these games will be evaluated by users - including healthy older adults and fallers - in the design workshops. We also intend to incorporate the users' sketches and designs in the development of future games that we will deploy in the community during the RCT.

Prior to the RCT, the effectiveness of these games on improving adherence to exercise will be evaluated in the community through a pilot study. The results from this study well enable us enhance the quality of our software and hardware tools for the RCT that will follow.

\section{ACKNOWLEDGMENT}

I am grateful to Fiona Fairlie for her support in writing this paper. This project is funded by the Lifelong Health and
Wellbeing (LLHW): a cross-research council initiative in partnership with the UK health departments.

\section{REFERENCES}

[1] Australian Commission on Safety and Quality in Health Care, (2009). Preventing falls and harm from falls in older people: Best practice guidelines for Australian hospitals and residential aged care facilities. http://www.safetyandquality.gov.au/internet/safety/publishing.nsf/Conte nt/FallsGuidelines-AustRACF

[2] L. Baillie, "The Home Workshop," Home-Oriented Informatics and Telematics International Working Conference, California, USA, 05/05/2003-10/05/2003.

[3] S. Benford, B. D. Bederson, K. P. Akesson, V. Bayon, A. Druin, P. Hansson, J. P. Hourcade, R. Ingram, H. Neale, C. O’Malley, K. T. Simsarian, D. Stanton, Y. Sundblad and G. Taxen, "Designing Storytelling Technologies to Encourage Collaboration Between Young Children," Paper presented at CHI' 2000: The Future is Here.

[4] J. M. Carroll, M. B. Rosson, G. Chin and J. Koenemann, "Requirements Development in scenario-based design," IEEE Transactions on Software Engineering, 24(12), 1156-1170, 1998.

[5] J. Doyle, C. Bailey, B. Dromey and C. N. Scanaill, "BASE - An interactive technology solution to deliver balance and strength exercises to older adults," Pervasive Computing Technologies for Healthcare (PervasiveHealth), 4th International Conference on-NO PERMISSIONS , vol., no., pp.1-5, 22-25 March 2010.

[6] D. Fitzgerald, N. Trakarnratanakul, L. Dunne, B. Smyth, B. Caulfield, "Development and user evaluation of a virtual rehabilitation system for wobble board balance training," Conf Proc IEEE Eng Med Biol Soc. 2008; 2008:4194-8.

[7] J. L. Gabbard, D. Hix and J. Edward Swan, "User-Centered Design and Evaluation of Virtual Environments," IEEE Journal of Computer graphics and Applications, Volume 19, Issue 6, November 1999.

[8] J. Hausdorff, "Gait Dynamics, Fractals and Falls: Finding Meaning in the Stride-to-Stride Fluctuations of Human Walking," Hum Mov Sci, 2007 August; 26(4): 555-589.

[9] Health Education Authority, Older people and accidents; Fact Sheet 2, London: HE, 1999.

[10] G. Kwakkel, R. Van Peppen, R. C. Wagenaar, S. Wood Dauphinee, C. Richards, A. Ashburn, K. Miller, N. Lincoln, C. Partridge, I. Wellwood and P. Langhorne, "Effects of augmented exercise therapy time after stroke: a meta-analysis," Stroke 35, 2529-2539, 2004.

[11] S. R. Lord, D. G. Lloyd and K. Sek, "Sensori-motor Function, Gait Patterns and Falls in Community-dwelling women," Journal of Age and Ageing, 1996:25:292-299.

[12] R. H. McKim, "Experiences in Visual Thinking," Boston Mass: PWS Publishers, 1972.

[13] A. Newell, J. Arnott, J. Carmichael and M. Morgan, "Methodologies for Involving Older People in the Design Process," Universal Access in Human Computer Interaction, Coping with Diversity," Lecture Notes in Computer Science, 2007, Volume 4554/2007, 982-989.

[14] Silver Fit Soft Kinetic rehabilitation, 2010, http://www.silverfit.nl.

[15] D. A. Skelton and S. M. Dinan, "Exercise for falls management: Rationale for an exercise programme aimed at reducing postural instability," Journal of Physiotherapy Theory and Practice, 1999.

[16] D. Taylor and C. Stretton, "The Otago Exercise Program, An evidencebased approach to falls prevention for older adults living in the community," Journal of Primary Health Care, Volume 31, Number 6, 2004.

[17] J. Tiesel and J. Loviscach, "A Mobile Low-Cost Motion Capture System Based on Accelerometers," Advances in Visual Computing, Lecture Notes in Computer Science, 2006, Volume 4292/2006, 437-446. 